\title{
Transition from morphologic diagnosis to immunophenotypic diagnosis of acute leukemia-experience of establishing a new flow cytometry laboratory
}

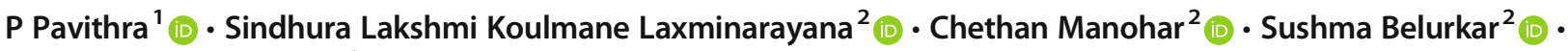 \\ Nikita Valerina Kairanna ${ }^{2}$ (D)
}

Received: 29 April 2019 / Accepted: 20 August 2019/Published online: 30 August 2019

(C) Springer-Verlag GmbH Germany, part of Springer Nature 2019

\begin{abstract}
In this era of targeted therapy, traditional reporting of acute leukemia by morphology using French-American-British (FAB) system of classification has limited uses due to lack of standardization and use in risk stratification. Flow cytometry (FCM), cytogenetics, and molecular testing drive the therapeutic decision. However, the lack of high-end testing at all health care strata leaves a general pathologist in a quandary. This study aimed at documenting the leukemia-associated immunophenotype (LAIP) specific for morphologic (FAB) subclasses of acute leukemia (AL). A retrospective case record-based study was carried out including 100 cases of de novo acute leukemia over 1 year to study the association of FCM immunophenotype profile with morphologic FAB classification of acute leukemia. Fourteen cases (14\%) were diagnosed as acute leukemia - unclassified by morphology which were accurately classified by FCM into B cell acute lymphoblastic leukemia (ALL) (6), T cell ALL (3), acute myeloid leukemia (AML) (4), and mixed phenotype acute leukemia (MPAL) (1). FCM also differentiated Pre B cell ALL from Burkitt lymphoma, subclassified T cell ALL into thymic categories, diagnosed MPAL, and identified blasts with monocytic differentiation which were not detected by morphology. Although morphologic FAB diagnosis is still widely used to classify acute leukemia, it is imperative that FCM should be used for accurate leukemia diagnosis.
\end{abstract}

Keywords Flow cytometry · French-American-British classification · Acute leukemia - Immunophenotyping · Leukemia-associated immunophenotyping

\section{Introduction}

Acute leukemia (AL) is a clonal hematopoietic stem cell disorder characterized by increase in blasts $(\geq 20 \%)$ in peripheral blood $(\mathrm{PB})$ and/or bone marrow $(\mathrm{BM})$ except in cases where specific cytogenetic abnormalities like $\mathrm{t}(8 ; 21)$

Sindhura Lakshmi Koulmane Laxminarayana

Sindhura.lakshmi@manipal.edu

P Pavithra

Pavithra.p@manipal.edu

1 Department of Pathology, Melaka Manipal Medical College, Manipal Academy of Higher Education, Manipal, Udupi, Karnataka 576104, India

2 Department of Pathology, Kasturba Medical College, Manipal, Manipal Academy of Higher Education, Manipal, Udupi, Karnataka 576104, India and inv(16) are present [1]. For nearly three decades, the diagnosis of AL relied heavily on French-American-British (FAB) system of classification based on morphology and enzyme cytochemistry for lineage assignment of blasts [2]. However, it fails to identify subclasses with poor prognosis except in acute promyelocytic leukemia and Burkitt lymphoma/leukemia. Also, there is a significant discordance in diagnosis given by different laboratories, sometimes within the same laboratory due to the subjective nature of morphologic reporting and lack of standardization of cytochemistry tests [3]. The diagnosis of AL depends on the examination by experienced observers of well-prepared $\mathrm{PB}$ and BM. This is particularly difficult in patients with nonleukemic conditions such as immune thrombocytopenia and lymphoma where an increase in normal B cell progenitors (hematogones) is found in bone marrow. These are virtually indistinguishable from blasts by morphology. Assignment of lineage is critical in the diagnosis of $\mathrm{AL}$ as it directs the therapeutic decision. Flow cytometry 
(FCM) immunophenotyping provides valuable information regarding leukemia-associated immunophenotype (LAIP), an insight into prognosis, and risk stratification. The 2008/2016 World Health Organization (WHO) classification of $\mathrm{AL}$ has included chromosomal and molecular features, in addition to morphology, immunophenotyping, and clinical manifestations [1]. However, this facility is not readily accessible to most hospitals in developing countries where diagnosis and classification of AL is still based on FAB system. The transition from morphologic diagnosis to immunophenotypic with further expansion to molecular diagnosis has not been easy. Several hurdles in terms of technical training, skill development, instrumentation, and providing a cost-effective, timely, and accurate reporting of acute leukemia have been faced by laboratories, which began an expanded diagnostic panel for acute leukemia. This study aims at documenting the LAIP specific for morphologic FAB subclasses of $\mathrm{AL}$ as reported during this period of transition.

\section{Materials and methods}

A retrospective record-based study was conducted in the hematology and clinical pathology laboratory of Kasturba Hospital, Manipal, after approval from the institutional ethics committee. Until the end of 2014, the laboratory did not have its own flow cytometer. The cases where morphologic diagnosis of AL was given were advised to undergo immunophenotyping, cytogenetic, and molecular testing. Hence, there were a huge number of "lost to follow-up" cases due to lack of adequate diagnosis. With the procurement of an eight-color, three-laser flow cytometer, instrument optimization, panel designing and standardization, technical training and skill development workshops were held in 2015. Thus, from the year 2016 onwards, a six-color antibody panel for acute leukemia diagnosis was fully functional.

\section{Establishment of minimal panel of antibodies}

Table 1 shows the antibody panel used in this study. An unstained lysed whole blood/bone marrow tube was included in each experiment. The antibody panel was adapted from the proceedings of a basic flow cytometry workshop held under the aegis of P.D. Hindja Hospital and Research Center, Mumbai, along with BD Biosciences, India. Titration of antibodies, antibody cocktail preparation, validation of the panels, and standardization of tests with the definition of positive and negative LAIP for blood and bone marrow were done under the guidance of an eminent alumnus (an experienced hematopathologist from one of the premier institutes in India) who kindly agreed to assist her alma mater.

\section{Training of personnel}

Initially, an in-house wet workshop and hands-on training was conducted by application executives from BD Biosciences for pathologists and technologists. The reporting pathologists also underwent training at flow cytometry laboratory of National Center for Biological Sciences, Bengaluru. They also took an active part in Indo-US flow cytometry workshops and annual meeting of the cytometry society-India for continued medical education. Membership of the cytometry society-India provided timely networking and mentoring support. Two technologists also received training at Tata Memorial Centre Advanced Centre for Treatment, Research and Education in Cancer, Navi Mumbai. This has now enabled our center to participate in inter-laboratory comparison program initiated by the institute.

\section{Case selection}

A total of 100 de novo AL cases diagnosed from January to December 2016 were included in the study. Cases with blast crisis secondary to chronic myeloid leukemia, mixed phenotype acute leukemia, follow-up acute leukemia patients on chemotherapy and post-transplant and cases of relapse were excluded from the study.

\section{Morphological examination}

Morphology was assessed on air-dried Leishman stained PB or BM aspirate smears and trephine biopsy sections stained by hematoxylin and eosin. All samples were stained with myeloperoxidase (MPO), periodic acid Schiff (PAS), and Sudan black B (SBB) cytochemical stains. Oil red O staining was done when Burkitt lymphoma was suspected. Acid phosphatase and non-specific esterase stains were not performed as the assay could not be standardized due to excessive background staining.

Reporting criteria by morphology were according to FAB classification [2].

\section{Immunophenotyping by FCM}

FCM was performed on PB/BM samples within $24 \mathrm{~h}$ of collection. Cytometer settings were tracked daily using a cytometer setting and tracking beads. Instrument optimization was done as per standard laboratory procedure, and the compensation matrix was linked to each experiment.

The samples were processed by the standardized "lysestain-wash" protocol for staining for surface markers. Lysestain-perm-stain-wash protocol was employed for simultaneous staining of surface and cytoplasmic markers. Each sample was processed in such a way that each tube had a cell concentration of one million cells per $100 \mu$ l. The cells were 
Table 1 Six-color antibody panel used for flow cytometric immunophenotyping of acute leukemia

\begin{tabular}{ll} 
Primary panel for Acute leukemia & \\
Tube 1 (B tube) & CD20, CD10, CD38, CD19, CD34, CD45 \\
Tube 2 (T tube) & CD8, sCD3, CD4, CD7, CD56, CD45 \\
Tube 3 (myeloid tube) & CD13, CD33, CD117, \\
& HLA DR, CD64, CD45 \\
Tube 4 (cytoplasmic tube) & MPO, CD79a, cCD3, CD34, CD45 \\
Secondary panel for Acute leukemia & \\
Tube 5 (cytoplasmic tube 2) & TdT, cCD22, cCD3, cCD41, CD45 \\
Tube 6 (T tube 2) & TCR, CD3, CD5, CD1a \\
Tube 7 (monocytic) & CD11c, CD14, CD33, CD36, CD45 \\
\hline
\end{tabular}

$C D$, cluster differentiation; $M P O$, myeloperoxidase; $H L A D R$, human leucocyte antigen-DR; TdT, terminal deoxynucleotidyl transferase stained with the six-color antibody panel in 4 primary tubes. Additional tubes were processed and run on a case to case basis depending on the results of primary panel (Table 1). The fluorochromes to which the monoclonal antibodies were conjugated included fluorescein isothiocyanate (FITC), phycoerythrin (PE), allophycocyanine (APC), peridinin chlorophyll protein cyanin 5.5 (PerCP Cy5.5), APC H7, and PE Cyanin 7 (PE Cy 7). The prepared specimen was acquired in a BD FACS CANTO II flow cytometer. All reagents and equipment were obtained from Becton Dickinson Biosciences, USA.

\section{Analysis of FCM data}

They were classified as acute myeloid or lymphoblastic leukemias based on the WHO 2008/2016 criteria for lineage assignment of blasts [1]. Blasts were gated using cluster differentiation (CD)45 and side scatter area (SSC), after doublet discrimination and excluding debris based on light scatter characteristics. Events with dim to moderate expression of CD45 with low SSC were considered as blasts which were further gated using CD19 (B lymphoblasts), cytoplasmic CD3 (T lymphoblasts), and cytoplasmic MPO (myeloblasts). B cell lineage was assigned when the blasts expressed CD19 brightly with more than one of the following: cytoplasmic CD79a, CD10, or CD20. In suspected cases of T cell ALL, events with bright CD45 expression and low SSC were gated as blasts and further analyzed for cytoplasmic CD3 and CD7 expression. T cell lineage was assigned if cytoplasmic CD3 or surface CD3 was positive. Events with intermediate to high SSC and moderate expression of CD45, negative CD34, and HLADR and bright MPO were considered as abnormal promyelocytes. Events with intermediate SSC and bright CD45 expression and positive for at least two monocytic markers (out of CD11c, CD14, CD36, CD64, or CD4 heterogeneous expression) were considered as blast equivalents of monocytic lineage (promonocytes and monoblasts). Events with no CD45 expression and low SSC were gated as possible proerythroblasts or megakaryoblasts. They were further evaluated for positive CD235A and CD41a respectively. Other markers were assessed for LAIP including lineage infidel (cross-lineage) antigen expression. LAIP was defined as follows [4]:

(1) asynchronous antigen expression (simultaneous expression of early and late markers in a single population such as the coexpression of CD34 and CD15 or CD19 and CD10);

(2) lineage infidelity, which is the expression of lymphoidassociated markers (CD3, CD5, CD7, CD56, CD10, and CD19) on myeloid blast cells; or myeloid associated antigens (CD13, CD33, or CD117);

(3) antigen overexpression, which is abnormally increased expression of a certain antigen per cell such as CD34, HLADR, and MPO in myeloblasts;

(4) absence of lineage-specific antigens, which involves absence of expected antigen expression, such as CD13 and CD33, on myeloid blasts and CD5 on T lymphoblasts

A surface marker was considered positive when $>20 \%$ of gated events showed positive expression. A cytoplasmic marker was considered positive when $>10 \%$ of gated events showed positive expression. The blasts were considered positive for cMPO when $>3 \%$ of gated events were positive. In the case of blasts which showed negative cMPO but positive for other myeloid associated antigens like CD13, CD33, and/or CD117, the results were confirmed by immunohistochemistry.

Demographic details such as age and gender were tabulated. $\mathrm{PB}$ and $\mathrm{BM}$ counts, morphologic diagnosis, immunophenotypic profile, and FCM diagnosis were recorded.

\section{Results}

During this 1-year retrospective study, a total of 100 cases of AL were included. Male:female ratio was 1.1:1. There were 51 cases 
$(51 \%)$ of acute lymphoblastic leukemia (ALL), acute myeloid leukemia (AML) was diagnosed in 46 cases (46\%), and 3 cases (3\%) were mixed phenotypic acute leukemia (MPAL) by FCM (Table 2). $60.7 \%$ of ALL patients were $<18$ years (mean age 18 years) while $56.5 \%$ patients with AML were $>50$ years (mean age 49.9 years). In $62 \%$ of cases, PB was used for FCM. In remaining cases, $\mathrm{BM}$ aspirate was analyzed usually due to low blast percentage in $\mathrm{PB}$. In these cases, mean PB blasts were $37.7 \%$ (overall PB blasts were 55.9\%). Morphologically, fourteen $(14 \%)$ cases were classified as AL without further subclassification as all special stains were negative in blasts and they could not be assigned a specific FAB subclass. FCM categorized them into B cell ALL (6), T cell ALL (3), AML (4), and MPAL (1) along with sub-classification (Table 3).

Out of 51 cases of ALL, 40 (78.4\%) were B cell ALL and 11 (21.5\%) were T cell ALL. All B cell ALL cases were positive for CD19, of which 36 (90\%) were CD10 positive pre B cell ALL. Cytoplasmic CD79a was positive in 37 (92.5\%) cases and CD20 was positive in $18(42.5 \%)$ cases. Six $(35.3 \%)$ cases of CD20 positive B cell ALL were negative for CD34, but all were dim CD45 positive and CD10 positive. Five of them lacked morphologic features of Burkitt lymphoma, hence, finally classified as pre B cell ALL. One case had large blasts with dense chromatin, round nuclei, and a vacuolated moderate amount of cytoplasm suggestive of FAB L3 morphology. However, oil red O was negative. Hence, a diagnosis of Pre B cell ALL was given and evaluation for MYC translocation was advised. Regrettably, the patient was lost to follow-up and further testing was not possible.
Out of 17 cases designated as $\mathrm{AL}$, nine cases were $\mathrm{CD} 10$ positive $\mathrm{B}$ cell ALL, and one was CD10 negative ALL as diagnosed by FCM. Thus, the use of FCM helped in identifying a poor prognostic marker in ALL, apart from accurate lineage assignment.

There were 11 cases (27.5\%) of T cell ALL, all of which all were CD7 positive. The most common markers for T ALL were cytoplasmic CD3 (91.7\%) and CD5 (83.3\%). There was a single case of T cell ALL, where both cytoplasmic and surface $\mathrm{CD} 3$ were negative. It was positive for CD7, $\mathrm{CD} 2$, and CD5 (small population) and negative for CD56, CD1a, CD8, and CD4. The blasts also expressed CD33 and HLA DR. It was classified as early T cell precursor ALL. The blasts were large and bizarre, with deep nuclear clefts and condensed chromatin with cytoplasmic vacuoles and blebs. Cytochemistry was negative for Oil Red stain, but occasional blasts showed block positivity for PAS. Another case with $\mathrm{CD} 7, \mathrm{cCD} 3$, and strong $\mathrm{CD} 5$ expression along with $\mathrm{CD} 117$ and HLADR was classified as near ETP-ALL. Interestingly, both these cases were diagnosed as AL without further classification by morphology as the blast morphology was not specific of any subtype and cytochemistry was negative for MPO, PAS, and SBBs. There were two cases of Pro T cell ALL (CD7+, cCD3+, and $\mathrm{sCD} 3 \pm, \mathrm{CD} 5 \pm, \mathrm{CD} 34+, \mathrm{TdT} \pm$, $\mathrm{HLADR} \pm$ ). In both these cases, blasts were small to medium in size, with dense chromatin, irregular cleaved nuclear contours, inconspicuous nucleoli, and scant pale cytoplasm (FAB L2). There were 2 cases of cortical T cell ALL (CD1a+, cCD3+, CD2+, CD5+, CD7+, CD4/CD8 double-positive)
Table 2 Case distribution by flow cytometry and morphologic diagnosis

\begin{tabular}{|c|c|c|c|c|}
\hline \multicolumn{5}{|c|}{ Total number of cases $(n)=100$} \\
\hline \multicolumn{3}{|c|}{ Flow cytometry diagnosis } & \multicolumn{2}{|l|}{ Morphologic diagnosis } \\
\hline ALL & & 51 & Acute leukemia unclassified & 14 \\
\hline$B$ cell $A L L$ & & 40 & ALL (FAB) & 42 \\
\hline T cell ALL & & 11 & L1 or L2 morphology & 41 \\
\hline$T$ & - ETP-ALL & 02 & To rule out L3 & 01 \\
\hline \multirow[t]{5}{*}{ cell $A L L$} & - Pro TALL & 02 & AML (FAB) & 44 \\
\hline & - Pre TALL & 01 & MO & 03 \\
\hline & - Cortical ALL & 02 & $M 1$ & 10 \\
\hline & - Late cortical ALL & 03 & $M 2$ & 09 \\
\hline & - Medullary & 01 & M3 & 04 \\
\hline \multicolumn{2}{|l|}{ AML } & 46 & M4 & 09 \\
\hline \multicolumn{2}{|l|}{$A M L N O S$} & 12 & $M 5 a$ & 04 \\
\hline \multicolumn{2}{|l|}{$A P M L$} & 03 & $M 5 b$ & 03 \\
\hline \multicolumn{2}{|c|}{ AML with monocytic differentiation } & 31 & M6 & 02 \\
\hline \multicolumn{2}{|l|}{ MPAL } & 03 & $M 7$ & 0 \\
\hline \multicolumn{2}{|l|}{ B/Myeloid } & 01 & & \\
\hline \multicolumn{2}{|l|}{ T/Myeloid } & $02^{*}$ & & \\
\hline
\end{tabular}

$F A B$, French-American-British classification; $A L L$, acute lymphoblastic leukemia; ETP, early T cell precursor; $A M L$, acute myeloid leukemia; NOS, not otherwise specified; $M P A L$, mixed phenotype acute leukemia.

Boldface entries indicate the type of leukemia and italic entries indicate different subtypes.

* These cases were diagnosed as AML by morphology 
Table 3 Summary of reclassification of cases by flow cytometry and their clinical course

Number of cases diagnosed as acute leukemia unclassified by morphology $=14$

\begin{tabular}{ll}
\hline Flow cytometry diagnosis & Number of cases \\
\hline B cell ALL & 06 \\
AML with monocytic differentiation & 04 \\
ETP-ALL & 02 \\
Medullary T cell ALL & 01 \\
B/myeloid MPAL & 01
\end{tabular}

$A L L$, acute lymphoblastic leukemia; $E T P$, early T cell precursor; $A M L$, acute myeloid leukemia, mixed phenotype acute leukemia

and 3 cases of late cortical T cell ALL (cortical phenotype with either CD4 or CD8 positivity). These blasts were small to medium in size, with scant cytoplasm and condensed chromatin and no nucleoli (FAB L1). A single case of medullary $\mathrm{T}$ cell ALL (cCD3, CD2, CD5, CD7, CD4, or CD8, positive and $\mathrm{CD} 1$ a negative) was diagnosed where blasts were of FAB L1 morphology.

There were 44 cases of AML diagnosed on morphology out of which three (6.8\%) cases were classified as FAB M0, 10 (22.7\%) FAB M1, 4 (9\%) FAB M3, 9 (20.4\%) each of FAB M2 and M4, 7 (15.9\%) FAB M5, and 2 (4.5\%) FAB M6. There were no cases of FAB M7. Three $(6.8 \%)$ cases showed marked myelodysplasia-related changes. One case each of FAB M2 and FAB M6 also showed significant dysplasiarelated changes. One case with FAB M1 had arisen in a preexisting primary myelofibrosis. A case of hypocellular AML FAB M2 was also noted. MPO cytochemistry was performed in 40/44 cases, where 3 cases were negative ( 2 cases of FAB $\mathrm{M} 0$ and a case of $\mathrm{AL}$ ) and 12 cases showed weak positivity (3-10\% of blasts, one case each of FAB M1 and M2, 6 cases of FAB M4, and 4 cases of FAB M5). Blasts in the remaining cases showed bright MPO positivity. Use of FCM reclassified 2/44 cases as T/Myeloid MPAL. Four out of 14 cases of acute leukemia unclassified were found to be AML by FCM. Thus, there were 46 cases of AML diagnosed by FCM (Table 2).

The concordance rate between cytochemistry and FCM for MPO was $94.8 \%$. One case (FAB M0) with negative MPO by cytochemistry was found positive for MPO by FCM. Another case (FAB M4) with weak MPO positivity was, in fact, negative by FCM. In both these discordant cases, results of FCM were confirmed by immunohistochemistry on bone marrow biopsy. The most common immunophenotypic markers used for AML diagnosis were CD33 (93.5\%), MPO and CD117 (86.9\%), and CD13 (82.6\%). Acute promyelocytic leukemia (FAB M3) was diagnosed in four (9\%) cases by morphology. While three of these cases were bright MPO+, CD13+, CD33+, CD117+, CD34- and HLA DR-; one case was $\mathrm{MPO}+, \mathrm{CD} 13+, \mathrm{CD} 33+, \mathrm{CD} 117+, \mathrm{CD} 64+$, and CD36+ suggestive of AML with monocytic differentiation. A total of 31 cases $(63.3 \%)$ expressed 2 or more monocytic lineage antigens. CD4 was positive in $25(43.5 \%)$ cases of which 21 (84\%) were also positive for CD64. In remaining cases, one or more of other monocytic markers (CD36, CD14, or CD11c) were positive. It is notable that only $12(48 \%)$ of these cases were classified as FAB M4 or M5 by morphologic examination. Out of 14 cases of unclassified AL, three cases turned out to be AML with monocytic differentiation by FCM. Thus, use of FCM was useful in identifying the monocytic immunophenotype across the FAB subclasses.

\section{LAIPs and number of cases expressing them is depicted in Table 4}

Three cases of MPAL diagnosed (two cases of T/myeloid and case of B/myeloid) based on WHO criteria for lineage assignment. These cases were classified as FAB M1, M2, and unclassified AL respectively.

\section{Discussion}

FCM in India has grown in log scale in the past decade. It started with few centers in metropolitan cities having flow cytometers to several centers across the country including smaller towns. There is no formal flow cytometry training available for pathology residents/medical lab technology students. There are superspeciality/fellowship courses in hematopathology in select few centers. This has created a gap between current global standards of FCM reporting and actual availability of skilled pathologists and technologists to work in FCM. It is imperative that the peripheral institutes must find a way to train the existing pathologists/ technologists in FCM to provide standardized and quality lab services. Indian Council for Medical Research has published basic guidelines on FCM in 2016 [5]. There is no national EQA program for FCM in India. An effort to run an inter-laboratory comparison program (ILCP) is underway. Yet, the demand for capacity building endeavors far exceeds these resources.

Although FAB classification is cheap and technically simple, subjective variation, lack of standardization, and inability to store diagnostic material for reevaluation limits its role in acute leukemia diagnosis. Immunophenotyping by FCM allows accurate identification of blasts, lineage assignment, and identification of therapeutic targets, prognostication, and minimal residual disease assay. Multiple antigen expression patterns may be studied in different subpopulations simultaneously, thus aids in the diagnosis of mixed phenotype acute leukemia [6].

Both PB and BM aspirate samples were found to be suitable for FCM, although BM aspirate samples were preferred 
Table 4 LAIP in different categories of acute leukemia. The numbers in the parenthesis indicate the number of cases

\begin{tabular}{|c|c|c|c|c|c|}
\hline & $\begin{array}{l}\text { Lineage-specific antigen } \\
\text { expression }\end{array}$ & $\begin{array}{l}\text { Asynchronous antigen } \\
\text { expression }\end{array}$ & Lineage infidelity & Antigen overexpression & $\begin{array}{l}\text { Antigen } \\
\text { underexpression }\end{array}$ \\
\hline B cell ALL & $\begin{array}{l}\text { CD19 (51) } \\
\text { c CD79A (37) }\end{array}$ & $\begin{array}{l}\text { CD10 (36) } \\
\text { CD34 (31) } \\
\text { HLADR (40) }\end{array}$ & $\begin{array}{l}\text { CD13 (04) } \\
\text { CD33 (02) }\end{array}$ & CD20 (18) & - \\
\hline T cell ALL & $\begin{array}{l}\text { c CD3 (10) } \\
\text { CD3 (04) } \\
\text { CD7 (11) }\end{array}$ & $\begin{array}{l}\text { CD4 and CD8 (1) } \\
\text { CD34 (04) } \\
\text { HLADR (02) }\end{array}$ & $\begin{array}{l}\text { c CD79a (02) } \\
\text { CD117 (01) } \\
\text { CD13 (01) } \\
\text { CD33 (01) }\end{array}$ & CD1a (05) & CD5 (09) \\
\hline AML & $\begin{array}{l}\text { CD33 (43) } \\
\text { c MPO (40) } \\
\text { CD13 (39) }\end{array}$ & $\begin{array}{l}\text { CD117 (40) } \\
\text { CD34 (33) } \\
\text { HLADR (38) }\end{array}$ & $\begin{array}{l}\text { CD7 (09) } \\
\text { CD19 (04) }\end{array}$ & - & - \\
\hline
\end{tabular}

$A L L$, acute lymphoblastic leukemia; $A M L$, acute myeloid leukemia; $C D$, cluster differentiation; $M P O$, myeloperoxidase; $H L A D R$, human leucocyte antigen - DR; LAIP, leukemia-associated immunophenotype

when PB blasts were less than $40 \%$. However, in cases where the provisional lineage assignment and subcategorization was critical as in case of APML, peripheral blood was analyzed for FCM despite lower blast count in the sample. In such cases, bulk lysis method was employed to improve the cell yield.

ALL was more common in children and AML in adults. ALs show a slight male preponderance. The overall occurrence of ALL was more than AML. These findings are comparable with other studies $[7,8]$.

In addition to FSC/SSC characteristics, dim to moderate expression of CD45 was used to gate the blasts. T lymphoblasts had a brighter expression of CD45. In AML with monocytic differentiation, which was classified as AML M4/M5 by morphology, the abnormal cells had a brighter expression of CD45 and higher FSC/SSC. Abnormal promyelocytes had similar side scatter as the granulocytes present in the specimen along with moderate expression of CD45. This approach helped in differentiating blast cell heterogeneity within a single sample as was reported in previous studies [9].

Literature review shows that the most common positive markers for AML were CD13 (95\%), CD33 (96\%), and CD117 (89\%); for B-ALL, CD19 (100\%), CD10 (82\%), CD79a and (95\%); for T ALL, c CD3 (100\%) and CD7 (100\%) [10]. The present study confirms the same.

B cell ALL was considerably more frequent than T cell ALL, and the majority were positive for CD10. Other studies have reported similar results $[11,12]$. In a study by Vitale et al., early T ALL (pre T \& pro T) was the most common subtype $(51 \%)$, followed by cortical $(39 \%)$ and medullary $(10 \%)$ [13].

Out of 100 cases, accurate lineage assignment was not possible in $14(14 \%)$. This was mainly because of nonspecific morphology of blasts, MPO negativity, or weak positivity by cytochemistry and presence of mixed phenotype blasts. FCM was able to correctly assign lineage to these blasts
(Table 4) and was indispensable in the diagnosis of MPAL. The cases where MPO was only weakly positive by cytochemistry, FCM was useful in confirming the diagnosis.

There was no definite correlation between FAB subtypes of ALL and the immunophenotypic diagnosis. In turn, FCM diagnosis provided valuable information regarding therapeutic targets such as CD19 and CD20 in B cell ALL and CD117 in AML. It helped in categorizing CD10 negative B cell ALL, identified acute promyelocytic leukemia (APML) accurately, and classified T cell ALL into respective groups of thymic differentiation. This was useful in advising specific cytogenetic and molecular studies, thereby reducing unnecessary use of large panel of high end, costly tests.

CD20 is a B cell differentiation antigen expressed in variable intensities in B lymphoblasts. The significance of CD20 expression in ALL is two-fold. Anti-CD20-directed immunotherapy by (rituximab, ofatumomab, and obinutuzumab) may significantly improve the clinical outcome in cases of newly diagnosed CD20 positive B cell ALL. Monotherapy with Blinatumomab is found to be useful in refractory and relapsed cases [14]. However, there is insufficient data in this regard from Indian subcontinent [15]. Expression of CD20 in a Philadelphia chromosome-negative B cell ALL is found to predict worse prognosis [16]. A population of large abnormal cells with bright CD45+, CD19+, CD20+, and cCD79a+ with monoclonal light chain expression and negative for blastic markers like CD34 and TdT must be evaluated for Burkitt lymphoma (BL). High Ki-67 index (nearly 100\%) and cmyc gene translocation are required for the diagnosis of BL. BL cells express strong surface IgM with light chain restriction, Bcl-6, CD19, CD20, CD22, PAX5, CD10, and CD79a, and are negative for $\mathrm{CD} 5, \mathrm{CD} 23$, and TdT. By contrast, precursor B cell ALL is usually TdT-positive and does not express surface immunoglobulin [1]. However, a small group (about $2 \%$ ) of pediatric BL with $\mathrm{t}(8 ; 14)(\mathrm{q} 24 ; \mathrm{q} 32)$ or variant 
MYC translocation express precursor B cell phenotype (TdT+ , CD34 \pm , CD20-, surface immunoglobulin negative). The reason for this variation is unknown and diagnosis must be given with caution [17]. In the present study, there were six cases of CD19+, cCD79a+, CD10+, CD20+, and CD34immunophenotype. All cases had dim CD45 expression and none had high Ki-67 index on immunohistochemistry, thus ruling out Burkitt lymphoma.

Apart from anti CD20 directed therapy, other novel molecular targets like CD19 (coltuximab ravtansine, denintuzumab) and CD22 (epratuzumab, inotuzumab ozogamicin, moxetumomab pasudolox) are under research. Anti CD52 therapy with alemtuzumab has been approved by US FDA in 2014 for refractory chronic lymphocytic leukemia but has not been promising for ALL therapy [14]. Thus, use of FCM for diagnosis of acute leukemia helps in identifying molecular targets for therapy.

CD10 is expressed by most of the acute lymphoblastic leukemias, certain lymphoid malignancies, and normal lymphoid progenitors. They are known to occur commonly in infants under 1 year of age, with marked leukocytosis $\left(>1 \times 10^{5} / \mu \mathrm{l}\right)$, central nervous system involvement and organomegaly. CD10 negative B cell ALLs are associated with rearrangements of mixed lineage leukemia (MLL) gene at 11q23. FISH using a dual-color break-apart probe is a useful technique to detect the MLL gene translocation [18]. In the present study, three cases of B cell ALL were CALLA negative. However, the cytogenetic and molecular testing was not done in these cases due to financial constraints.

There was no significant correlation between FAB subtypes and T cell ALL. Out of 11 cases, 3 cases showed block granular positivity in PAS stain. The historical description of T lymphoblast having deeply convoluted nuclei and scant cytoplasm was found to be non-specific as such blasts were found in B cell ALL as well. The immunophenotype assisted in categorizing the Precursor T cell ALL into subclasses based on their normal thymic counterparts. Although such categorization helped in understanding the $\mathrm{T}$ cell ontogeny, studies indicate that it does not have implications on clinical outcome [1].

Assessment of MPO by FCM allows confirmation of cytochemical diagnosis particularly in cases with weak expression. In the present study, MPO was positive $86.9 \%$ cases. In a study by Kim Y et al., $62 \%$ of cases were MPO positive. They were reported to be associated with better disease-free survival, and transplant was beneficial [19]. FCM is more sensitive in detecting blasts with monocytic differentiation (Table 5). Thirty-one cases were positive for 2 or more monocytic markers (CD64, CD14, CD36, CD11c, and CD4) by FCM whereas combined morphology and cytochemistry could diagnose only 16 cases. However, among these, there were two cases of FAB AML M6 and a case of AML with myelodysplasia-related changes as well. Our series shows much higher number of cases of AML with monocytic
Table 5 AML with monocytic differentiation diagnosed by FCM. Expression of twos or more markers of monocytic differentiation was considered as diagnostic criterion for this entity

\begin{tabular}{|c|c|c|c|c|c|c|}
\hline \multirow{2}{*}{$\begin{array}{l}\text { Morphologic } \\
\text { diagnosis by } \\
\text { FAB }\end{array}$} & \multirow{2}{*}{$\begin{array}{l}\text { Number of } \\
\text { cases of AML } \\
\text { with monocytic } \\
\text { differentiation } \\
\text { by FCM }\end{array}$} & \multicolumn{5}{|c|}{$\begin{array}{l}\text { Number of cases positive for markers } \\
\text { of monocytic differentiation }\end{array}$} \\
\hline & & CD64 & CD14 & CD11c & CD36 & CD4 \\
\hline AL-unclassified & 4 & $1 / 4$ & $1 / 4$ & $1 / 4$ & $2 / 4$ & $4 / 4$ \\
\hline M0 & 0 & 0 & 0 & 0 & 0 & 0 \\
\hline M1 & 6 & $2 / 6$ & $3 / 6$ & $4 / 6$ & $3 / 6$ & $6 / 6$ \\
\hline M2 & 5 & $1 / 5$ & $1 / 5$ & $1 / 5$ & $5 / 5$ & $5 / 5$ \\
\hline M3 & 1 & $1 / 1$ & $0 / 1$ & $0 / 1$ & $0 / 1$ & $1 / 1$ \\
\hline M4 & 6 & $4 / 6$ & $5 / 6$ & $6 / 6$ & $3 / 6$ & $6 / 6$ \\
\hline M5a & 4 & $4 / 4$ & $4 / 4$ & $4 / 4$ & $4 / 4$ & $4 / 4$ \\
\hline M5b & 3 & $2 / 3$ & $0 / 3$ & $2 / 3$ & $2 / 3$ & $3 / 3$ \\
\hline M6 & 2 & $2 / 2$ & $2 / 2$ & $2 / 2$ & $2 / 2$ & $2 / 2$ \\
\hline M7 & 0 & 0 & 0 & 0 & 0 & 0 \\
\hline
\end{tabular}

$F A B$, French-American-British classification; $A M L$, acute myeloid leukemia; FCM, flow cytometry; $C D$, cluster differentiation; $A L$, acute leukemia

differentiation when compared with other Indian studies who have reported $28 \%$ [6] to $8.6 \%$ [15] of such cases by FCM. Such variation is probably due to differences in the FCM antibody panel that is used in different centers. Hence, the final diagnosis of AML with monocytic leukemia must not be made based on immunophenotype alone. Instead, morphologic features should be considered in conjunction with FCM diagnosis. Diagnosis of APML by FCM utilizes multiple aberrant antigen expression patterns. An abnormal cell population with high SSC, dim to moderate CD45 expression with bright MPO, CD13, and CD33 expression and negative CD34 and HLADR, clinches the diagnosis. Additionally, overexpression of CD2 and underexpression of CD15 and CD9 also help in diagnosis. Morphologically, hypogranular variant of APML with high peripheral blood blast $\%$ is a close mimic of monoblastic/monocytic leukemia. Although cytochemical stains like MPO (strong positive) and non-specific esterase (negative) help in differentiating these two entities, FCM has proven to be more objective. In our study, out of 4 morphological APMLs, one case turned out to be AML with monocytic differentiation by FCM.

Mixed phenotypic acute leukemias (MPAL) are a heterogeneous group of rare leukemias where the blasts exhibit lineage-specific antigens of more than one lineage [20]. Earlier reports from India have reported an incidence of 2$3 \%[21,22]$. This is in accordance with our study (3\%). It is not possible to detect MPAL without flow cytometry as they do not exhibit any specific morphologic features. In the present study, all three cases were classified as AML by morphology. An accurate diagnosis of MPAL is critical as these 
patients are more likely to benefit from ALL directed therapy than being treated for AML which might prove too toxic and lead to early mortality [23].

The present is based on a 6-color panel of antibodies directed towards a fairly broad array of antigens. All cases of acute leukemia demonstrated one or more LAIP (Table 3). Lineage-specific antigen expression helped in assigning lineage to blasts and in the diagnosis of MPAL. Asynchronous expression of antigens was the most common type of aberrancy. The pattern of such expression also provided a clue to abnormal maturation pattern and in some cases, an evidence of dyspoiesis. Lineage infidel aberrant antigen expression is defined as co-expression of markers usually not present on cells of that particular lineage such as lymphoid antigenpositive AML and myeloid antigen-positive ALL, not classified as MPAL. It is useful for prognostication and detection of minimal residual disease. Aberrant expression of CD56 in AML has been associated with extramedullary leukemia and correlates with reduced disease-free survival and overall survival [24]. In our study, $32 \%$ cases of AML had aberrant antigen expression. In this study, CD7 was the most common lymphoid-associated antigen expressed, followed by CD19. Similar findings were reported in earlier studies $[25,26]$. In $\mathrm{B}$ cell ALL, lineage infidel aberrant expression was seen in $20.51 \%$ cases; CD13 expression is the most common. Five out of $11 \mathrm{~T}$ cell ALL had aberrant B lymphoid and myeloid antigen expression. This is in contrast with earlier reports where such an expression was low [27]. It is possible to ascertain more lineage infidel aberrant antigen expression by using a comprehensive panel of antibodies for flow cytometry. Antigen overexpression and underexpression were seen in a few cases. There was no correlation between lineage infidel aberrant antigen expressions with FAB sub-classification. However, knowledge of such aberrancies at diagnosis helps in minimal/measurable residual disease assay by FCM where LAIP approach is used.

FCM was able to reclassify the acute leukemia unclassifiable cases (14 cases) as shown in Table 4 . When FCM facility was not available at our center, such cases were referred to centers where more advanced testing and therapeutic options were available. However, this resulted in high "lost to followup" cases and patients' inability to access such facilities due to financial constraints. Due to the availability of more accessible modes of definitive diagnosis, these patients were able to receive appropriate therapy.

\section{Conclusion}

Flow cytometry is an essential tool for diagnosis and subclassification of AL. It is useful for differentiating B and T cell ALLs, correct identification of AML- M0, MPO negative AML, MPAL, and aberrant markers, and in determining minimal residual disease (MRD). This will further aid in prognosis and in taking appropriate treatment decisions. The transition from morphology to immunophenotype diagnosis is only a small step towards precision medicine in the management of acute leukemia. Capacity building programs for peripheral laboratories are essential for standardized testing by FCM.

\section{Compliance with ethical standards}

The study was reviewed and approved by the institutional ethics committee at Kasturba Medical College, Manipal. All procedures followed were in accordance with the ethical standards of the responsible committee on human experimentation (institutional and national) and with the Helsinki Declaration of 1975, as revised in 2008.

Conflict of interest The authors declare that they have no conflicts of interest.

\section{References}

1. Arber DA, Orazi A, Hasserjian R et al (2016) The 2016 revision to the World Health Organization classification of myeloid neoplasms and acute leukemia. Blood 127:2391-2405

2. Bennett JM, Catovsky D, Daniel MT, Flandrin G, Galton DA, Gralnick HR, Sultan C (1976) Proposals for the classification of the acute leukaemias. French-American-British (FAB) cooperative group. Br J Haematol 33(4):451-458

3. Sengar M, Rai AK, Saxena A, Singh A, Raina V, Seth T, Sharma A, Bakhsi S, Kumar R, Mitra DK (2009) Acute leukemia: diagnosis improved by flow cytometry in addition to morphology. Asia Pac J Clin Oncol 5:55-65. https://doi.org/10.1111/j.1743-7563.2009. 01188

4. Al-Mawali A, Gillis D, Hissaria P et al (2008) Incidence, sensitivity, and specificity of leukemia-associated phenotypes in acute myeloid leukemia using specific five color multiparameter flow cytometry. Am J Clin Pathol 129(6):934-945

5. Gujral S, Kaur T. (2016) Standard operating procedures for immunophentyping of hematolymphoid neoplasms. ICMR. New Delhi

6. Koju S, Sachdeva MUS, Bose P, Varma N (2015) Spectrum of acute leukemias diagnosed on flow cytometry: analysis from tertiary care centre from North India. Ann Clin Chem Lab Med 1(1):12-15

7. Singh G, Parmar P, Kataria SP, Singh S, Sen R (2016) Spectrum of acute and chronic leukemia at a tertiary care hospital, Haryana, India. Int J Res Med Sci 4(4):1115-1118

8. Rego MF, Pinheiro GS, Metze K, Lorand-Metze I (2003) Acute leukemias in Piaui: comparison with features observed in other regions of Brazil. Braz J Med Biol Res 36(3):331-337

9. Lacombe F, Durrieu F, Briais A, Dumain P, Belloc F, Bascans E, Reiffers J, Boisseau MR, Bernard P (1997) Flow cytometry CD45 gating for immunophenotyping of acute myeloid leukemia. Leukemia. 11(11):1878-1886

10. Haycocks NG, Lawrence L, Cain JW, Zhao XF (2011) Optimizing antibody panels for efficient and cost effective flow cytometric diagnosis of acute leukemia. Cytom Part B-Clin Cy 80(B):221-229

11. Rajkumar NN, Vijay RH (2017) Immunological subtypes of acute lymphoblastic leukemia-beyond morphology: experience from Kidwai State Cancer Institute, Bengaluru, India. J Assoc Physicians India 65(7):14-17 
12. Hegde S, Soundarya P, Harsha K (2013) Study of the clinicopathological profile of paediatric haematological malignancies at a tertiary care centre. J Evol Med Dent Sci 2(43):8438-8443

13. Vitale A, Guarini A, Ariola C et al (2006) Adult T-cell acute lymphoblastic leukemia: biologic profile at presentation and correlation with response to induction treatment in patients enrolled in the GIMEMA LAL 0496 protocol. Blood. 107(2):473-479

14. Frahadfar N, Litszow MR (2016) New monoclonal antibodies for the treatment of acute lymphoblastic leukemia. Leuk Res 49:13-21. https://doi.org/10.1016/j.leukres.2016.07.009

15. Gupta N, Pawar R, Banerjee S, Brahma S, Rath A, Shewale S, Parihar M, Singh M, Arun SR, Krishnan S, Bhatacharyya A, Das A, Kumar J, Bhave S, Radhakrishnan V, Nair R, Chandy M, Arora N, Mishra D (2019) Spectrum and immunophenotypic profile of acute leukemia: a tertiary center flow cytometry experience. Mediterr J Hematol Infect Dis 11(1):e2019017. https://doi.org/10. 4084/mjhid.2019.017

16. Gokbuget N, Hoelzer D (2004) Treatment with monoclonal antibodies in acute lymphoblastic leukemia: current knowledge and future prospects. Ann Hematol 83:201-205

17. Navid F, Mosijczuk AD, Head DR, Borowitz MJ, Carroll AJ, Brandt JM, Link MP, Rozans MK, Thomas GA, Schwenn MR, Shields DJ, Vietti TJ, Pullen DJ (1999) Acute lymphoblastic leukemia with the $(8 ; 14)(\mathrm{q} 24 ; \mathrm{q} 32)$ translocation and FAB L3 morphology associated with a B-precursor immunophenotype: the pediatric oncology group experience. Leukemia. 13(1):135-141

18. Devi SG, Goyal M, Ramakrishna NVSS, Murthy SS (2011) CALLA negative precursor B lymphoblastic leukemia with MLL gene translocation and an unusual FISH signal pattern. Indian $\mathrm{J}$ Pathol Micr. 54(1):176-179

19. Kim Y, Yoon S, Jeong Kim S, Seok Kim J, Cheong JW, Min YH (2012) Myeloperoxidase expression in acute myeloid leukemia helps identifying patients to benefit from transplant. Yonsei Med J 53(3):530-536c

20. Weinberg OK, Arber DA (2010) Mixed-phenotype acute leukemia: historical overview and a new definition. Leukemia 24:1844-1851. https://doi.org/10.1038/leu.2010.202 published online 16 September 2010

21. Sukumaran R, Nair RA, Jacob PM, Nair Anila KR, Prem S, Binitha $R$ et al (2015) Flow cytometric analysis of mixed phenotype acute leukemia: experience from a tertiary oncology center. Indian J Pathol Micro 58:181-186

22. Sarma A, Sharma JD, Bhuyan C, Hazarika M, Kataki AC (2013) A biphenotypic (mixed phenotypic) acute leukemia: report of two cases with immunophenotypic study. Open J Blood Dis 3:65-68

23. Wolach O, Stone RM (2017) Mixed-phenotype acute leukemia: current challenges in diagnosis and therapy. Curr Opin Hematol 24(2):139-145. https://doi.org/10.1097/MOH.0000000000000322

24. Ossenkoppele G, Van de Loosdrecht A, Schuurhuis GJ (2011) Review of the relevance of aberrant antigen expression by flow cytometry in myeloid neoplasms. Br J Haematol 153:421-436

25. Xu B, Hu C, Miao X, et al. (2003) Immunophenotyping of 106 adult patients with acute leukemia by flow cytometry. Di Yi Jun Yi Da Xue Xue Bao 23:1043-6

26. Bahia D, Yamamoto M, Chauffaille M et al (2001) Aberrant phenotypes in acute myeloid leukemia: a high frequency and clinical significance. Haematologica. 86:801-806

27. Belurkar S, Kurien A, Manohar C, Mantravadi H (2013) Correlation of morphologic and cytochemical diagnosis with flow cytometric diagnosis of acute leukemia. J Cancer Res Ther 1:71-79

Publisher's note Springer Nature remains neutral with regard to jurisdictional claims in published maps and institutional affiliations. 